\section{OPEN ACCESS}

Edited by:

Robert Braidwood Sim University of Oxford, United Kingdom

Reviewed by:

Zoltan Prohaszka,

Semmelweis University, Hungary Christian Drouet,

INSERM U1016 Institut

Cochin, France

József Dobó,

Institute of Enzymology

(MTA), Hungary

*Correspondence:

Johannes Zschocke

johannes.zschocke@i-med.ac.at

Specialty section:

This article was submitted to

Molecular Innate Immunity,

a section of the journal

Frontiers in Immunology

Received: 30 July 2019

Accepted: 14 October 2019

Published: 05 November 2019

Citation:

Gröbner R, Kapferer-Seebacher I, Amberger A, Redolfi R, Dalonneau F, Björck E, Milnes D, Bally I, Rossi V,

Thielens N, Stoiber H, Gaboriaud C and Zschocke J (2019) C1R Mutations

Trigger Constitutive Complement 1 Activation in Periodontal Ehlers-Danlos Syndrome. Front. Immunol. 10:2537. doi: 10.3389/fimmu.2019.02537

\title{
C1R Mutations Trigger Constitutive Complement 1 Activation in Periodontal Ehlers-Danlos Syndrome
}

\author{
Rebekka Gröbner ${ }^{1}$, Ines Kapferer-Seebacher ${ }^{2}$, Albert Amberger ${ }^{1}$, Rita Redolfi ${ }^{1}$, \\ Fabien Dalonneau ${ }^{3}$, Erik Björck ${ }^{4,5}$, Di Milnes ${ }^{6}$, Isabelle Bally ${ }^{3}$, Veronique Rossi ${ }^{3}$, \\ Nicole Thielens ${ }^{3}$, Heribert Stoiber ${ }^{7}$, Christine Gaboriaud ${ }^{3}$ and Johannes Zschocke ${ }^{1 *}$ \\ 1 Institute for Human Genetics, Medical University Innsbruck, Innsbruck, Austria, ${ }^{2}$ Department for Operative and Restorative \\ Dentistry, Medical University Innsbruck, Innsbruck, Austria, ${ }^{3}$ University of Grenoble Alpes, CEA, CNRS, IBS, Grenoble, \\ France, ${ }^{4}$ Department of Molecular Medicine and Surgery, Karolinska Institute, Stockholm, Sweden, ${ }^{5}$ Department of Clinical \\ Genetics, Karolinska University Hospital, Stockholm, Sweden, ${ }^{6}$ Genetic Health Queensland, Royal Brisbane and Women's \\ Hospital, Herston, QLD, Australia, ${ }^{7}$ Institute of Virology, Medical University Innsbruck, Innsbruck, Austria
}

Heterozygous missense or in-frame insertion/deletion mutations in complement 1 subunits $\mathrm{C} 1 \mathrm{r}$ and $\mathrm{C} 1 \mathrm{~s}$ cause periodontal Ehlers-Danlos Syndrome (pEDS), a specific EDS subtype characterized by early severe periodontal destruction and connective tissue abnormalities like easy bruising, pretibial haemosiderotic plaques, and joint hypermobility. We report extensive functional studies of $16 C 1 R$ variants associated with pEDS by in-vitro overexpression studies in HEK293T cells followed by western blot, size exclusion chromatography and surface plasmon resonance analyses. Patient-derived skin fibroblasts were analyzed by western blot and Enzyme-linked Immunosorbent Assay (ELISA). Overexpression of C1R variants in HEK293T cells revealed that none of the pEDS variants was integrated into the $\mathrm{C} 1$ complex but cause extracellular presence of catalytic $\mathrm{C} 1 \mathrm{r} / \mathrm{C} 1 \mathrm{~s}$ activities. Variants showed domain-specific abnormalities of intracellular processing and secretion with preservation of serine protease function in the supernatant. In contrast to C1r wild type, and with the exception of a C1R missense variant disabling a C1q binding site, pEDS variants had different impact on the cell: retention of $\mathrm{C} 1 \mathrm{r}$ fragments inside the cell, secretion of aggregates, or a new $\mathrm{C} 1 \mathrm{r}$ cleavage site. Overexpression of C1R variants in HEK293T as well as western blot analyses of patient fibroblasts showed decreased levels of secreted C1r. Importantly, all available patient fibroblasts exhibited activated C1s and activation of externally added C4 in the supernatant while control cell lines secreted proenzyme C1s and showed no increase in C4 activation. The central elements in the pathogenesis of pEDS seem to be the intracellular activation of $\mathrm{C} 1 \mathrm{r}$ and/or $\mathrm{C} 1 \mathrm{~s}$, and extracellular presence of activated $\mathrm{C} 1 \mathrm{~s}$ that independently of microbial triggers can activate the classical complement cascade.

Keywords: complement system, connective tissue, periodontitis, C1r/s, Ehlers-Danlos syndrome

\section{INTRODUCTION}

Periodontitis is a common chronic disease caused by a dysbalance between the oral microbiome and the host immune response leading to inflammatory destruction of the tooth-supporting tissues and finally loss of teeth. Periodontal Ehlers-Danlos Syndrome (pEDS) (OMIM 130080 and 617174, prevalence: unknown) is an autosomal dominant type of Ehlers-Danlos Syndrome 
(EDS) (1, 2) characterized by early severe and rapidly progressing periodontal destruction and variable connective tissue abnormalities (3). Clinical features include lack of attached gingiva already present in childhood, easy bruising, pretibial haemosiderotic plaques, doughy skin, hoarse voice, poor wound healing, and hypermobility of joints. We recently showed that pEDS is caused by heterozygous missense or in-frame insertion/deletion mutations in C1R or C1S (4). These genes code for complement 1 subunits $\mathrm{C} 1 \mathrm{r}$ and $\mathrm{C} 1 \mathrm{~s}$, serine proteases that play a key role in the innate immune response. The penetrance in the individuals with pEDS identified up to now is $100 \%$, and there is no clinical evidence for relevant modifier genes.

$\mathrm{C} 1 \mathrm{r}$ and $\mathrm{C} 1 \mathrm{~s}$ have a similar protein domain structure with the N-terminal interaction domain including CUB (complement C1r/C1s, Uegf, Bmp1) and EGF (epidermal growth factor)

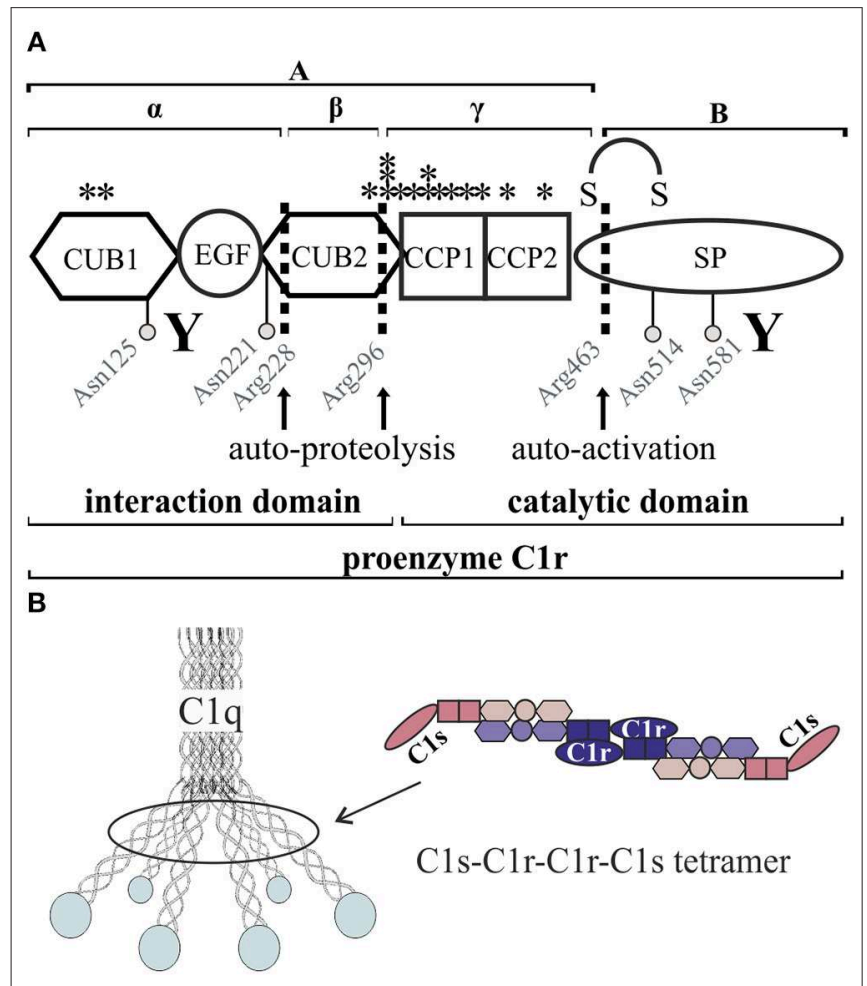

FIGURE 1 | Schematic overview of C1r domain structure and secretion pattern. (A) Cleavage sites (arrows) as well as glycosylation sites (gray circles) are marked. Investigated pEDS variants (see also Table 2) are marked with stars. CUB1-EGF-CUB2 is described as the interaction domain and CCP1-CCP2-SP as the catalytic domain of C1r. "Y" indicates $\mathrm{N}$ - and $\mathrm{C}$-terminal antibody target regions used in this study. Full-length proenzyme C1r has a molecular mass of $\sim 100 \mathrm{kDa}$ on western blot. Activation occurs through cleavage at Arg463, which produces the disulfide-linked A- and B-chains with apparent molecular masses when analyzed by SDS-PAGE under reducing conditions of 55 and $38 \mathrm{kDa}$. C1r is also known to undergo two additional auto-proteolytic cleavages at Arg 228 and 296 in the A-chain that produce an $\mathrm{N}$-terminal $\alpha$-fragment with an apparent mass of $35 \mathrm{kDa}$, a $\beta$-fragment, and a $\gamma$-fragment disulfide-linked to the B-chain $(5,6)$. The fragments $\beta$ and $\gamma$ cannot be detected under reducing conditions by the $\mathrm{C} 1 \mathrm{r}$ antibodies used in this study. (B) The $\mathrm{C} 1$ complex consists of a $\mathrm{C}_{1} \mathrm{r}_{2}-\mathrm{C} 1 \mathrm{~s}_{2}$ tetramer embedded into the umbrella-like hexamer of C1q heterotrimers. modules in a CUB1-EGF-CUB2 arrangement, two complementcontrol-protein modules CCP1 and CCP2, and a serine protease (SP) domain (Figure 1A, Table 1). Two molecules of C1r and $\mathrm{C} 1 \mathrm{~s}$ form a tetramer which binds to the collagen-like stalks of $\mathrm{Clq}$ assembled from six heterotrimers to build up the $\mathrm{C} 1$ complex (Figure 1B). Binding of the resulting C1 complex to activating targets such as antibody-antigen complexes causes $\mathrm{C} 1 \mathrm{q}$ conformation changes which trigger auto-activation of C1r. This involves cleavage of the protein between the Nterminal A-chain and the C-terminal B-chain; both chains remain linked through a disulfide bridge. C1r activation causes cleavage of $\mathrm{C} 1 \mathrm{~s}$ at a similar position, and subsequently activation of $\mathrm{C} 4$ and $\mathrm{C} 2$, ultimately resulting in activation of the central complement protein $\mathrm{C} 3(7,8)$. Activation of C3 and downstream signaling pathways triggered by host-microbe interactions has been shown to promote inflammatory bone loss in periodontitis (9). Based on the results from a C3-knock-out mouse model (10), C3-targeted drug candidates have been suggested as novel immunotherapeutics for periodontal disease (11). Overactivation of the complement system causes periodontitis as proposed by several pre-clinical studies and clinical case reports and was reviewed elsewhere (12).

Pathogenic $C 1 R$ and $C 1 S$ variants in pEDS are expected to be gain-of-function variants as heterozygous loss-offunction variants in these genes are asymptomatic while homozygous variants cause systemic lupus erythematosus without periodontitis (13-15). However, the exact gain of function mechanism has not yet been characterized. Homozygosity for pEDS causing variants has not been observed. All $C 1 R$ and $C 1 S$ pEDS variants identified so far are located in the A-chain, mostly CCP1 and CCP2. In order to elucidate the pathomechanism underlying pEDS we systematically investigated the molecular consequences of all $16 C 1 R$ variants known at the onset of the study.

\section{MATERIALS AND METHODS Patient-Derived Skin Fibroblasts and C4a ELISA}

Skin fibroblast samples were obtained after informed written consent from pEDS patients in four families with $C 1 R$ variants

TABLE 1 | Summary of all C1r fragments with their predicted and apparent mass.

\begin{tabular}{llccc}
\hline $\begin{array}{l}\text { Amino } \\
\text { acid }\end{array}$ & Description & $\begin{array}{c}\text { Predicted } \\
\text { polypeptide } \\
\text { chain mass } \\
\text { (kDa) }\end{array}$ & $\begin{array}{c}\text { Number of } \\
\text { N-linked } \\
\text { sugars }\end{array}$ & $\begin{array}{c}\text { Apparent mass } \\
\text { on } \\
\text { western blot } \\
\text { (kDa) }\end{array}$ \\
\hline $18-463$ & A (heavy) chain & 51 & 2 & 55 \\
$18-228$ & $\alpha$-fragment & 28 & 2 & 35 \\
$229-296$ & $\beta$-fragment & 7.7 & - & Not detected \\
$18-296$ & $\alpha \beta$-fragment & 35.7 & 2 & 40 \\
$297-449$ & $\gamma$-fragment & 19.6 & - & Not detected \\
$464-705$ & B (light) chain & 27 & 2 & 38 \\
$18 . . .705$ & Proenzyme C1r & 78 & 4 & 100
\end{tabular}


c.149_150TC $>$ AT (p.V50D; $n=3)$, c.277G > T (p.G93C; $n=1)$, c.902G >C (p.R301P; $n=2$ ), and c.926G > T (p.C309F; $n=1$ ). All pathogenic variants identified were specific for individual families, without evidence of recurrence for any pEDS variant. We did not identify any family with a de-novo mutation although parental samples were not available for all affected individuals. Fibroblasts from healthy individuals served as controls.

Fibroblasts were cultured at $37^{\circ} \mathrm{C} / 5 \% \quad \mathrm{CO}_{2}$ in DMEM (Sigma Aldrich Chemie GmbH, Schnelldorf, Germany, \#D5546500ML) supplemented with $10 \%$ fetal bovine serum (FBS) (Gibco, Fisher Scientific GmbH, Vienna, Austria, \#10500064), penicillin/streptromycin (Sigma Aldrich Chemie $\mathrm{GmbH}$, \#P4333), and 2 mM L-glutamine (Lonza, Szabo-Scandic, Vienna, Austria, \#BE17-605E). RNA was extracted with RNeasy Kit (Qiagen, Hilden, Germany, \#74104) according to manufacturer's instructions for validation of heterozygous expression of mutated C1R. RNA was reverse transcribed (Fermentas, Distributor: Fisher Scientific Austria GmbH, \#K1652) followed by PCR-based amplification of $C 1 R$ and Sanger sequencing.

Medium was exchanged from DMEM to Ex-Cell 293 serumfree cell culture medium for HEK293T cells (Sigma Aldrich Chemie GmbH, \#14571C-1000ML) supplemented with $2 \mathrm{mM} \mathrm{L-}$ glutamine $48-72 \mathrm{~h}$ prior to harvest of supernatant. Exchange to serum-free medium was necessary to prevent detection of serumderived complement proteins. Cells were harvested separately and lysed for $10 \mathrm{~min}$ on ice in RIPA buffer $(50 \mathrm{mM}$ Tris$\mathrm{HCl} \mathrm{pH} 7.4,0.154 \mathrm{M} \mathrm{NaCl}, 1 \mathrm{mM}$ EDTA, $0.5 \%$ Triton X-100, $0.05 \%$ SDS) containing protease inhibitor (Fisher Scientific Austria $\mathrm{GmbH}$, \#87786). Protein concentration of cell lysates was determined by Bradford assay (Biorad Laboratories $\mathrm{GmbH}$, Vienna, Austria, \#500-0006). Harvested cell culture supernatants were concentrated 10-fold with Pierce Protein Concentrator (Fisher Scientific Austria GmbH, \#88517). Protein concentration of cell culture supernatants was normalized based on the protein concentration of the respective cell lysates. Cells and supernatants were stored at $-20^{\circ} \mathrm{C}$ until further analyses.

Enzyme-linked Immunosorbent Assay (ELISA) based quantification of $\mathrm{C} 4 \mathrm{a}$ (Quidel, Distributor: Biomedica Medizinprodukte GmbH \& Co KG, Vienna, Austria, \#A036) concentrations was conducted with concentrated cell culture supernatants following the supplier's instructions. C4 purified from human serum (Sigma Aldrich, \#C8195) was added at a final concentration of $2.2 \mathrm{ng} / \mathrm{ml}$ to harvested cell culture supernatant. Fibroblasts express C1r, C1s and C1 inhibitor (C1Inh) but not C1q or C4; qPCR analysis of fibroblasts showed no difference in the amounts of transcripts of C1R, C1S, and SERPING1 (coding for C1Inh) between patients and controls (data not shown). Standard curve as well as samples were measured as duplicates. Statistical analyses were performed with GraphPad Prism 7.00. Results were tested for normality with D-Agostino-Pearson omnibus normality test. Since not all sample groups passed the normality test, we applied the unpaired, non-parametric Mann-Whitney test (significance level $\alpha=0.05 ;{ }^{*} \leq 0.05 ;{ }^{* *} \leq$ 0.01 ; $\left.^{* *} \leq 0.001\right)$. C4a levels were determined in supernatant of cultured fibroblasts and serum from individuals with C1R c.149_150TC >AT (p.V50D), C1R c.902G >C (p.R301P), and $C 1 R$ c.926G $>$ T (p.C309F). Cell culture supernatant was harvested and stored as described above. Serum samples were immediately centrifuged and frozen to $-80^{\circ} \mathrm{C}$; there were no differences in sample handling between patients and controls.

\section{HEK293T Cells as in-vitro Overexpression Model of C1r Variants}

A total of 16 different $C 1 R$ variants from two publications $(4,16)$ were investigated, including one previously unpublished variant c. $277 \mathrm{G}>\mathrm{T}$ (p.G93C) identified in a family from Australia (Table 2). All variants are predicted to be pathogenic, and none of the variants has been found in registered control populations [ExAc database http://exac.broadinstitute.org/ (17), last accessed September 26, 2019]. Variant p.G93C is the only novel variant in the present study; it is not recorded in variant databases (e.g., GnomAD) and is predicted to be probably damaging by PolyPhen. In addition, we studied two missense variants recorded in control samples in the ExAc database. The mutation type and location could be compatible with a functional effect but both variants were predicted to be benign by variant assessment programs such as PolyPhen, and no association with pEDS was reported. One of the variants (c.547C $>$ T, p. (E183K)) has a very high minor allele frequency of 0.1174 , precluding a strong unknown functional effect, the other variant (c.902G $>$ A, p.(R301C)) is very rare (minor allele frequency 0.00006612 ) but is at the same position as a disease-causing variant identified by us in a pEDS patient. We expected that overexpression of these variants should be indistinguishable from wild type.

Finally, we generated enzymatically inactive C1r WT and variants by adding a previously designed active site mutation p.S654A to WT and variant clones (18). This variant removes the active center Serine residue which is essential for the serine protease function. Under physiological conditions in plasma, proenzyme activation of normal $\mathrm{C} 1 \mathrm{r}$ is prevented by presence of C1Inh. Variant p.S654A precludes auto-activation, also in the absence of C1Inh. It is not known whether p.S654A interferes with C1Inh; however, this is irrelevant with regard to the loss of function effect of the variant.

C1R WT was cloned into the transfection vector pcDNA3.1(+)NEO (GenScript, Distributor: Hölzel Diagnostika Handels GmbH, Köln, Germany), and all variants were inserted by site-directed mutagenesis (Peqlab, Distributor: VWR, Vienna, Austria, \#07-KK2100-01) according to manufacturer's instructions. C1s WT was cloned into a p3xFLAG-CMV14 vector. HEK293T cells (Sigma Aldrich Chemie $\mathrm{GmbH}$, Schnelldorf, Germany) were cultured at $37^{\circ} \mathrm{C} / 5 \% \quad \mathrm{CO}_{2}$ in EMEM (Sigma Aldrich Chemie GmbH, \#M2279-500 ML) supplemented with $10 \%$ FBS, penicillin/streptomycin and $2 \mathrm{mM}$ L-glutamine. For transfection purposes, $1 \times 10^{6}$ cells were seeded into T-25 flasks the day before transfection. Immediately before transfection, cells were washed with PBS and medium was exchanged to EMEM without FBS. Transfection was conducted with $12 \mu \mathrm{l}$ Turbofect (Fisher Scientific GmbH, \#R0531) and 4 $\mu \mathrm{g}$ of $C 1 R$ plasmid in $1 \mathrm{ml}$ EMEM. Five hours after transfection cell medium was exchanged to EMEM supplemented with FBS and penicillin/streptomycin. The day after transfection, medium was exchanged to Ex-Cell 293 serum-free culture 
TABLE 2 | List of all C1R variants analyzed in this study.

\begin{tabular}{|c|c|c|c|c|c|}
\hline DNA (c.) (GRCh38) & Protein (p.) & Domain & MAF & $\begin{array}{l}\text { PolyPhen/*PROVEAN } \\
\text { prediction }\end{array}$ & ClinVar \\
\hline c.149_150TC>AT & p.Val50Asp & CUB1 & Not listed & prob.dam (1.000) & VCV000372130 \\
\hline c. $277 \mathrm{G}>\mathrm{T}$ & p.Gly93Cys & CUB1 & Not listed & prob.dam (0.986) & VCV000597277 \\
\hline c. $869 A>G$ & p.(Asp290Gly) & C1q-binding & Not listed & prob.dam (0.987) & VCV000267356 \\
\hline c. $890 \mathrm{G}>\mathrm{A}$ & p.(Gly297Asp) & CUB2 & Not listed & prob.dam (1.000) & VCV000372129 \\
\hline c.899T>C & p.(Leu300Pro) & CUB2 & Not listed & prob.dam (0.997) & VCV000267354 \\
\hline c. $902 \mathrm{G}>\mathrm{C}$ & p.Arg301Pro & CUB2 & Not listed & poss.dam (0.719) & VCV000267352 \\
\hline c. $905 A>G$ & p.(Tyr302Cys) & CUB2 & Not listed & prob.dam (1.000) & VCV000375577 \\
\hline c.917_927delinsGGACA & p.(Ile306_Cys309delinsArgArg) & CCP1 & Not listed & deleterious ${ }^{\star}(-15.383)$ & VCV000267355 \\
\hline c. $927 \mathrm{C}>\mathrm{G}$ & p.(Cys309Trp) & CCP1 & Not listed & prob.dam (1.000) & VCV000267353 \\
\hline c. $926 \mathrm{G}>\mathrm{T}$ & p.Cys309Phe & CCP1 & Not listed & prob.dam (0.996) & Not available \\
\hline c. $1012 \mathrm{~T}>\mathrm{C}$ & p.(Cys338Arg) & CCP1 & Not listed & prob.dam (1.000) & VCV000375578 \\
\hline c. $1073 G>T$ & p.(Cys358Phe) & CCP1 & Not listed & prob.dam (1.000) & VCV000267351 \\
\hline c. $1092 \mathrm{G}>\mathrm{C}$ & p.(Trp364Cys) & CCP1 & Not listed & prob.dam (1.000) & VCV000375579 \\
\hline c. $1113 \mathrm{C}>\mathrm{G}$ & p.(Cys371Trp) & CCP1 & Not listed & prob.dam (1.000) & VCV000375580 \\
\hline c.1200_1215delinsTCATGTAATA & p.(Arg401_Tyr405delinsHisVallle) & CCP2 & Not listed & deleterious ${ }^{*}(-19.321)$ & VCV000375581 \\
\hline c. $1303 \mathrm{~T}>\mathrm{C}$ & p.Trp435Arg & CCP2 & Not listed & prob.dam (1.000) & VCV000375582 \\
\hline c.547C > T & p.(Glu183Lys) & EGF-like & 0.1174 & benign (0.006) & Not available \\
\hline c. $902 \mathrm{G}>\mathrm{A}$ & p.(Arg301Cys) & CUB2 & 0.00006612 & benign (0.397) & Not available \\
\hline
\end{tabular}

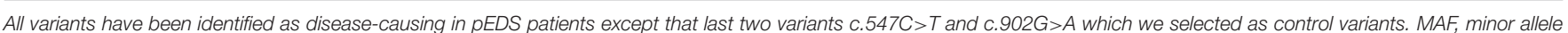
frequency (ExAc database). "*” indicates prediction with PROVEAN Protein tool (http://provean.jcvi.org/index.php). prob.dam, probably damaging; poss.dam, possibly damaging.

medium supplemented with $2 \mathrm{mM}$ L-glutamine. Cells and supernatants were harvested $48 \mathrm{~h}$ after transfection, as described for patient-derived skin fibroblasts.

In order to determine the ability of C1r to cleave $\mathrm{C} 1 \mathrm{~s}$, concentrated cell culture supernatants of single transfected HEK293T cells containing C1r (WT or variant) were mixed 1:1 with supernatant of $\mathrm{C} 1 \mathrm{~s}$ transfected cells. Supernatant mixtures were incubated for $1 \mathrm{~h}$ at $37^{\circ} \mathrm{C}$ and analyzed by western blot (C1s antibody).

\section{Western Blot Analyses}

Western blot analyses were performed under denaturing and reducing conditions (unless indicated otherwise) using standard procedures. Protein samples were separated by using a $10 \%$ SDS gel (Biorad Laboratories $\mathrm{GmbH}$, \#4561031) and running buffer (20 mM Tris, $0.2 \mathrm{M}$ glycine, $3.5 \mathrm{mM}$ SDS). Twenty microgram of whole cell lysate were used and supernatant normalized to according cell pellet. Page Ruler prestained protein ladder (Fisher Scientific GmbH, \#26616) was used for determining protein molecular weight. Proteins were transferred onto a PVDF membrane via wet blotting $\left(1 \mathrm{~h}, 100 \mathrm{~V}, 4^{\circ} \mathrm{C}\right)$. Membranes were probed with $\mathrm{C} 1 \mathrm{r}$ or $\mathrm{C} 1 \mathrm{~s}$ specific antibodies (Abcam plc, Cambridge, UK; C1r: N-terminal \#ab71652 or Cterminal \#ab185212; C1s: \#ab155270) diluted 1:2,000 in PBS0.05\% Tween 20 (PBS-T) containing 5\% milk (w/v). HRPconjugated secondary antibody (Dako, Glostrup, Denmark, \#P0448) was used 1:10,000 in 5\% milk/PBS-T. Protein bands were detected with ECL (GE Healthcare, Vienna, Austria, \#RPN3243) and developed on X-ray films (Amersham Hyperfilm ECL, Distributor: VWR, Vienna, Austria). For overexpressed C1r variants, empty vector transfected HEK293T cells were used as negative control.

\section{Size Exclusion Chromatography of the C1s-C1r-C1r-C1s Tetramer}

Proenzyme C1s-C1r-C1r-C1s tetramers containing C1r WT or variant with additional p.S654A mutation were produced by co-transfected 293-F cells and purified as described (19). Size exclusion chromatography of the purified tetramers was performed by using a Superose 6 Increase 10/300 GL column (GE Healthcare, UGAP, Marne la Vallée, France) equilibrated in $50 \mathrm{mM}$ Tris, $150 \mathrm{mM} \mathrm{NaCl}, \mathrm{pH} 7.4$ containing $2 \mathrm{mM} \mathrm{CaCl}_{2}$ or $5 \mathrm{mM}$ EDTA at a flow rate of $0.5 \mathrm{ml} / \mathrm{min}$.

\section{Surface Plasmon Resonance (SPR) Analyses}

Binding of the $\mathrm{C} 1 \mathrm{r}-\mathrm{C} 1 \mathrm{~s}$ tetramer to immobilized $\mathrm{C} 1 \mathrm{q}$ was analyzed by SPR using a Biacore T200 instrument (GE Healthcare). Plasma derived C1q [purified according to Arlaud et al. (20)] was diluted at $35 \mu \mathrm{g} / \mathrm{ml}$ in $10 \mathrm{mM}$ sodium acetate $\mathrm{pH} 5$ and immobilized on a Series S CM5 sensor chip (GE Healthcare) using the amine coupling chemistry in $10 \mathrm{mM}$ Hepes, $150 \mathrm{mM}$ $\mathrm{NaCl}$, EDTA 3 mM pH 7.4, surfactant P20 0.05\% (HBS-EP+, GE Healthcare). A flow cell submitted to the coupling steps without immobilized protein was used as blank. Binding of the tetramer was measured by injecting $90 \mu \mathrm{l}$ of each tetramer $(5 \mathrm{nM})$ at a flow rate of $30 \mu \mathrm{l} / \mathrm{min}$ in $50 \mathrm{mM}$ Tris- $\mathrm{HCl}, 150 \mathrm{mM} \mathrm{NaCl}, 2 \mathrm{mM}$ $\mathrm{CaCl}_{2}, 0.05 \%$ Tween 20, pH 7.4 followed by 300 s dissociation. The specific binding signal was obtained by subtracting the signal 
over the blank surface. Regeneration was achieved by a $15 \mu \mathrm{l}$ injection of $1 \mathrm{M} \mathrm{NaCl}, 10 \mathrm{mM}$ EDTA, pH 7.4.

\section{Production and Protein Fragment Analysis of the p.W435R Variant of $\mathrm{C} 1 \mathrm{r}$}

Recombinant p.W435R variant of $\mathrm{C} 1 \mathrm{r}$ was produced in the FreeStyle 293 Expression System (Thermo Fisher), using a pcDNA3.1/Neo(+) plasmid encoding human $\mathrm{C} 1 \mathrm{r}$ with a C-terminal Strep-tag. 293-F cells grown in FreeStyle 293 medium were transfected with this plasmid using 293 fectin and stable transfectants were selected with $400 \mu \mathrm{g} / \mathrm{ml}$ neomycin (Thermo Fisher). Recombinant C1r was purified from the culture supernatant by chromatography on StrepTactin Sepharose High performance (Sigma-GE28-9355-99), as recommended by the manufacturer. Briefly, the cell culture supernatant was concentrated 6-fold, dialyzed against $100 \mathrm{mM}$ Tris, $150 \mathrm{mM}$ $\mathrm{NaCl}, 1 \mathrm{mM}$ EDTA, $\mathrm{pH} 8.0$, and applied to a 1-ml column equilibrated in the same buffer. Elution was carried out using $2.5 \mathrm{mM}$ desthiobiotin (Sigma-Aldrich) in the same buffer. The eluted protein was dialyzed in the same buffer and concentrated to $0.3-0.45 \mathrm{mg} / \mathrm{ml}$. N-terminal sequence determination of the major band observed after SDS-PAGE analysis under nonreducing conditions was performed using an Applied Biosystems gas-phase sequencer model 492 coupled online with an Applied Biosystems Model 140C HPLC system.

\section{RESULTS}

\section{pEDS Variants Cause Domain-Specific Abnormalities of C1r Processing and Secretion}

Intracellular processing and secretion of $\mathrm{C} 1 \mathrm{r} \mathrm{WT}$ and 16 different $\mathrm{C} 1 \mathrm{r}$ variants (Table 2) was studied by transient overexpression in HEK293T cells (which do not express C1r/s or C1q) using western blot analysis of cell lysates and supernatants. The results obtained by an antibody against the $\mathrm{N}$-terminal domain of $\mathrm{C} 1 \mathrm{r}$ are depicted in Figure 2. Because of auto-activation in the absence of $\mathrm{C} 1$ inhibitor (C1Inh; Gene: SERPING1), little full-length $\mathrm{C} 1 \mathrm{r}(100 \mathrm{kDa})$ but presence of A-chain was detected in all cell lysates. Variants resulted in intracellular presence of A-chain, sometimes with an additional smaller C1r fragment that may represent a degradation product or reduced glycosylation. Overexpressed C1r WT is effectively secreted into the supernatant where it is visible either as the auto-activated A-chain $(55 \mathrm{kDa})$, or the $\alpha$-fragment $(35 \mathrm{kDa})$ generated via an auto-proteolytic event $(5,6)$. The same WT activation and secretion pattern was observed for the two control variants p.(R301C) and p.(E183K), as expected. In contrast, all pEDSrelated C1r variants except C1r p.(D290G) showed evidence of intracellular accumulation as shown by an increased intracellular signal of activated C1r A-chain, and abnormal secretion patterns as follows (Figure 2):

1. The N-terminal anti-C1r antibody showed strongly reduced signals for CUB1 variants p.V50D (no bands) and p.G93C (reduced A-chain, absent $\alpha$-fragment) in the supernatant under standard conditions. Increased loading volume and exposure time during detection revealed traces of A-chain and $\alpha$-fragment also for p.V50D.

2. Variants in the CUB2 domain (except p.(D290G)) resulted in two fuzzy bands of molecular weight higher than WT (42 $\mathrm{kDa}$ and $100 \mathrm{kDa}$, respectively) that may represent aggregates formed by C1r A-chains. These fragments were not visualized by a C-terminal anti-C1r antibody, confirming that they did not represent full-length $\mathrm{C} 1 \mathrm{r}$.

3. Secretion of C1r with the CUB2 p.(D290G) variant was similar to C1r WT. Size exclusion chromatography showed that C1r WT and p.(D290G) yielded similar major peaks in the presence of $\mathrm{CaCl} 2$ indicating that variant proteins form C1s-C1r-C1r-C1s tetramer like C1r WT (Figure 3A). In the presence of EDTA, the tetramer was dissociated into two later eluting peaks, corresponding to the $\mathrm{C} 1 \mathrm{r}$ dimer and $\mathrm{C} 1$ s monomer. However, SPR analyses revealed that the C1r p.(D290G) variant strongly inhibits binding of the tetramer to $\mathrm{C} 1 \mathrm{q}$ and forming of a functional $\mathrm{C} 1$ complex (Figure 3B). Similar results were observed previously for the C1r p.D290A variant (21).

4. CCP1 variants were absent in the supernatant under normal conditions. Increased exposure time revealed traces of abnormal fragments (comparable to pattern 2) that did not represent full-length when detected by a C-terminal antibody.

5. CCP2 variants yielded an $\mathrm{N}$-terminal fragment $(45 \mathrm{kDa})$ slightly shorter than the A-chain that does not give rise to an $\alpha$-fragment and does not seem to undergo auto-proteolytic cleavage. In order to determine the origin of the shorter fragments found for these variants, the recombinant p.W435R variant was overexpressed, partially purified and $\mathrm{N}$-terminally sequenced. This identified a new cleavage site at Arg401 of CCP2 leading to an abnormal A-chain shortened by 62 amino acids. The 3D structure shows proximity between p.W435 and the new cleavage site (Figure 3C). The two cleavage sites correspond to C1r specificity (cleavage between Arg and Ile).

\section{Pathogenic C1r Variants Are Associated With Secretion of Active Serine Protease}

Supernatants of representative variants from each domain were investigated in more detail under reducing and nonreducing conditions using specific $\mathrm{N}$ - and $\mathrm{C}$-terminal anti-C1r antibodies (Figure $\mathbf{4}$ and Figure S1). These analyses confirmed the previously observed secretion patterns under reducing conditions (Figure 4A). The B-chain, detected by the C-terminal antibody was present in the supernatants of C1r WT and all $\mathrm{C} 1 \mathrm{r}$ variants studied (Figure 4B). The investigated CCP1 variant (p.(C338R)) gave a very weak signal in western blots in repeated analyses, indicating reduced or slow secretion of this variant. Under non-reducing western blot conditions (Figures 4C,D), full-length protein was strongly present in the supernatant for WT and variant p.(D290G). Smaller amounts of full-length protein were found for the CCP2 variant (p.W435R) that gives rise to an abnormal cleavage site (this was only shown by the C-terminal antibody which is more sensitive for full-length $\mathrm{C} 1 \mathrm{r}$ than the $\mathrm{N}$-terminal antibody, see also Figures $4 \mathrm{C}, \mathrm{D}$ ). The serine protease is present as B-chain (reducing conditions) or 


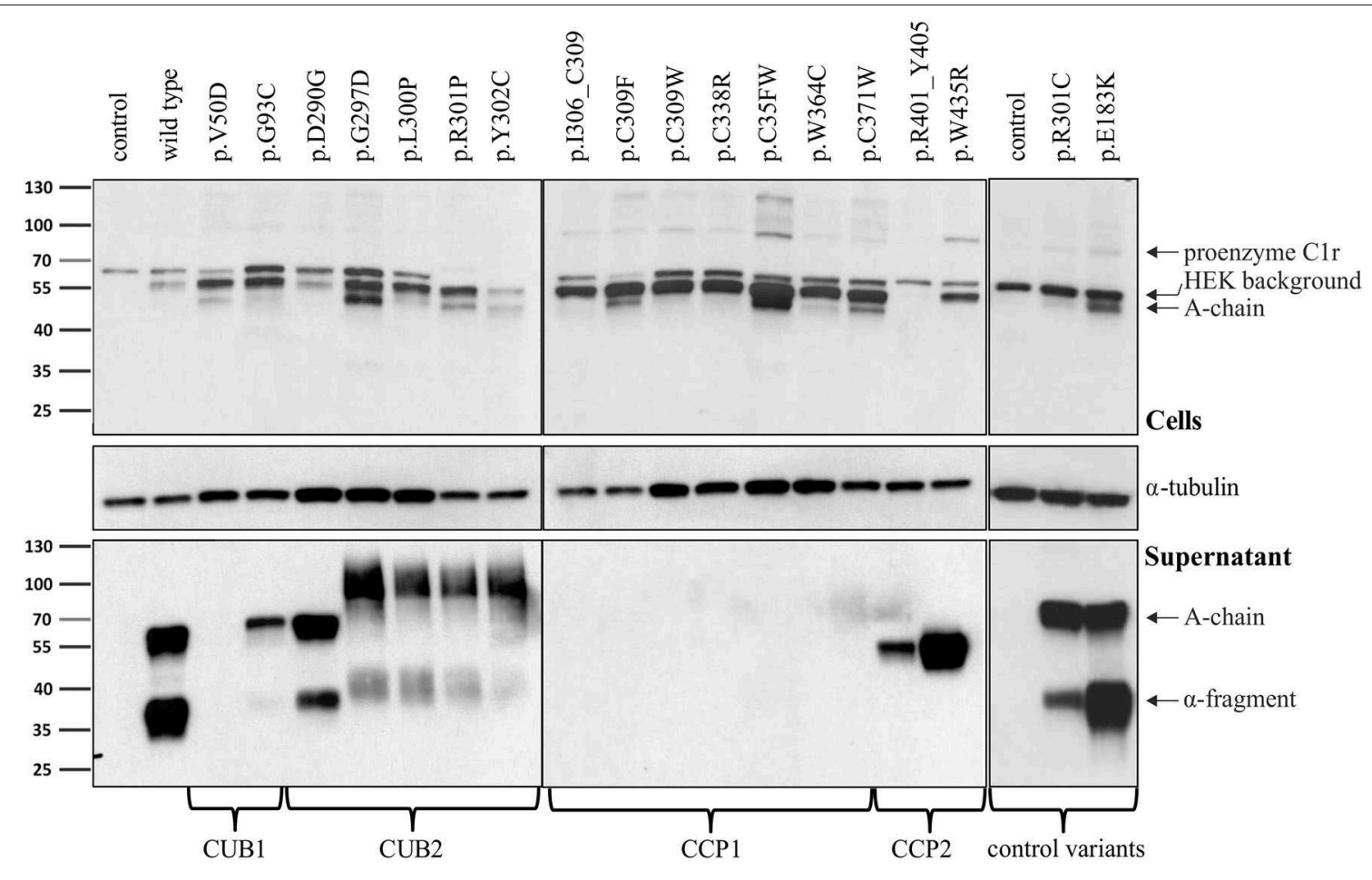

FIGURE 2 | Domain-specific secretion patterns of C1r variant proteins. HEK293T cells were transiently transfected with C1r WT or variants ("control" = empty vector). Cell lysates (upper panel) and supernatants (lower panel) were used for western blot with an N-terminal anti-C1r antibody $48 \mathrm{~h}$ after transfection under reducing conditions. This antibody may specifically visualize (1) the full-length $\mathrm{C} 1 \mathrm{r}(100 \mathrm{kDa})$; (2) the A-chain (55 kDa) after auto-activation of C1r; or (3) the $\alpha$-fragment (35 kDa) generated via an auto-proteolytic event after secretion of the activated protein. An intracellular HEK-specific background band is seen at around $60 \mathrm{kDa}$. Little full-length $\mathrm{C} 1 \mathrm{r}(100 \mathrm{kDa})$ and presence of A-chain was detected in all cell lysates. Most variants resulted in increased intracellular presence of A-chain fragments, sometimes with an additional smaller C1r fragment that may represent a degradation product. Overexpression of C1r WT, the control variants p.(R301C) and p.(E183K), and the variant p.(D290G), resulted in high amounts of A-chain and $\alpha$-fragment in the supernatant. All other variants show domain-specific abnormalities of intracellular processing and secretion. Strongly reduced extracellular secretion was found for variants in the CUB1 and CCP1 domains. All CUB2 variants (except p.(D290G)) show abnormal protein aggregates in the supernatant, the exact nature of which cannot be specified. Abnormal protein fragments without proteolysis were found for CCP2 variants.

$\gamma \mathrm{B}$-fragment (non-reducing) for all variants studied, although it was weak or absent for the CCP1 variant (p.(C338R)), and the $\gamma \mathrm{B}$-fragment was shortened for the CCP2 variant (p.W435R; predicted size: $34 \mathrm{kDa}$ ) because of the new cleavage site.

In the next step, mutation p.S654A was inserted in $\mathrm{C} 1 \mathrm{r}$ WT and all C1r variants. The mutation Ser654 to Ala destroys the active center of the serine protease domain by itself, independently of any interaction with C1Inh. Therefore, this mutation (p.S654A) prevents auto-activation and subsequent auto-proteolytic cleavage of C1r. In this setting, WT as well as variant p.(D290G) and the CCP2 variant (p.W435R) showed secretion of the non-activated full-length protein in western blots with both, $\mathrm{N}$ - and C-terminal specific anti-C1r antibodies. No C1r protein was detected for the other inactivated $\mathrm{C} 1 \mathrm{r}$ variants (CUB1, CUB2, CCP1) indicating intracellular retention or degradation (Figures 4E,F).

\section{All C1r Variants Retain Enzymatic Activity Toward C1s}

The only known substrate of C1r is C1s, which is cleaved by activated C1r. Thus, C1s cleavage by $\mathrm{C} 1 \mathrm{r}$ WT and variants was determined by two different experimental settings.
Co-incubation of C1s (WT) and C1r (WT or variant) supernatant showed that all analyzed C1r variants cleaved $\mathrm{C} 1 \mathrm{~s}$, whereas uncleaved full-length $\mathrm{C} 1 \mathrm{~s}$ was present when $\mathrm{C} 1 \mathrm{~s}$ supernatant was incubated in absence of C1r (Figure 5A). Cleavage is demonstrated by the presence of the C1s A-chain in western blots. Insertion of the inactivating mutation p.S654A into all C1r constructs prevents C1s cleavage (Figure 5B), confirming that $\mathrm{C} 1 \mathrm{~s}$ activation was due to $\mathrm{C} 1 \mathrm{r}$ activity and not to an unspecific protease activity in the supernatants.

In a second approach, C1s was co-expressed with C1r WT or variants in HEK293T cells. Results shown in Figure 5C demonstrate $\mathrm{C} 1 \mathrm{~s}$ activation in all co-transfection experiments regardless of C1r WT or mutation status. C1s cleavage (albeit partial) was observed in cell lysates, indicating that $\mathrm{C} 1 \mathrm{~s}$ activation may occur during post-translational processing within the cell.

\section{Constitutive C1s Activation in Fibroblasts From Individuals With Periodontal EDS}

Individuals with pEDS caused by heterozygous $C 1 R$ variants co-express WT and variant transcripts in equal amounts, and also co-express $\mathrm{C} 1 \mathrm{~s}$ and $\mathrm{C} 1 \mathrm{Inh}$. Fibroblasts from five individuals with pEDS (three different patients with the same variant 

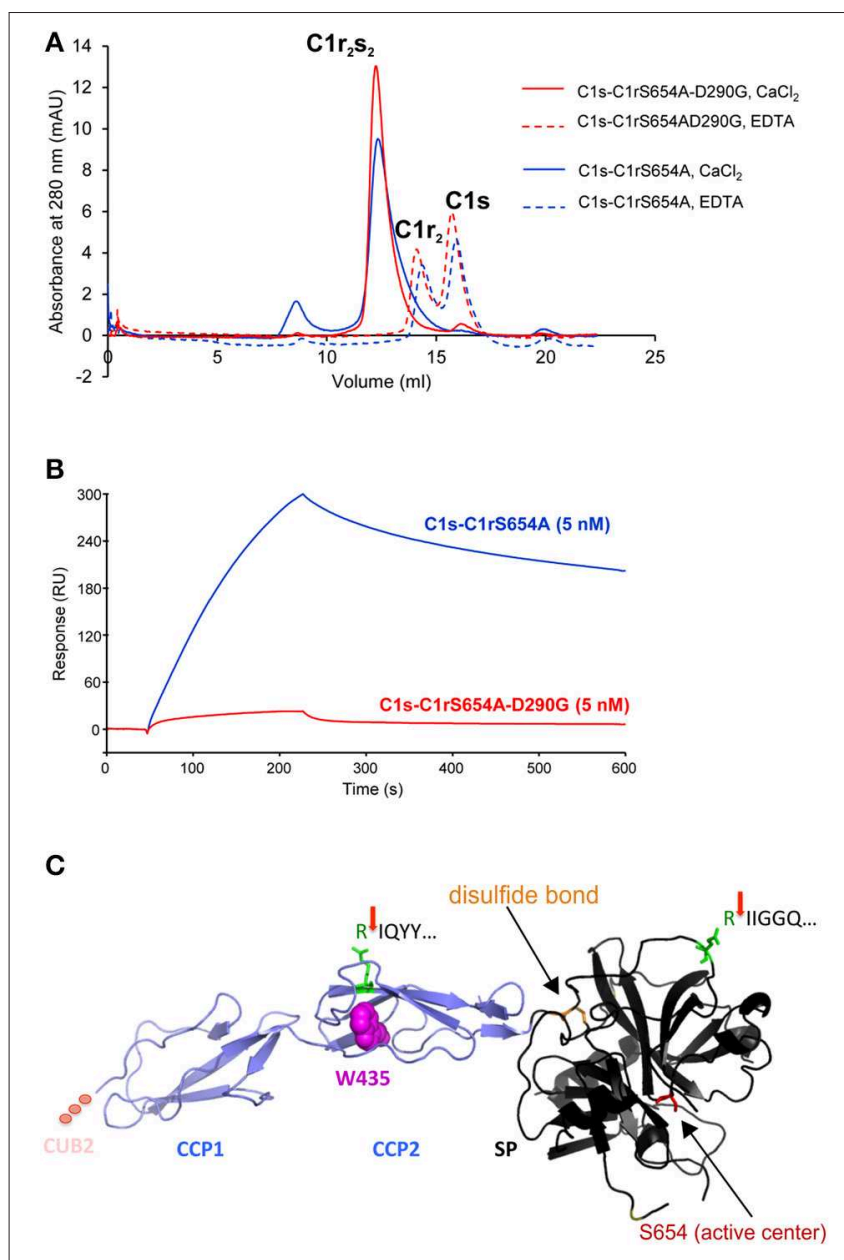

FIGURE 3 | C1r p.(D290G) variant strongly inhibits binding of the tetramer to C1q whereas C1r p.W435R induces a new cleavage site. (A) Size exclusion chromatography of the tetramers (containing C1r p.S654A with/without p.(D290G)) in the presence of $\mathrm{CaCl}_{2}$ (plain lines) or EDTA (dotted lines). WT and variant $\mathrm{C} 1 \mathrm{r}$ yielded similar major peaks in the presence of $\mathrm{CaCl}_{2}$. In the presence of EDTA, the tetramer was dissociated into two later eluting peaks, corresponding to the C1r dimer and C1s monomer. (B) SPR analysis of the binding of the tetramers to immobilized C1q (14,300 RU). Ninety microliter of each tetramer $(5 \mathrm{nM})$ were injected at a flow rate of $30 \mu \mathrm{l} / \mathrm{min}$ followed by 300 s dissociation. C1r p.(D290G) allows formation of the C1s-C1r-C1r-C1s tetramer but strongly inhibits binding of the tetramer to $\mathrm{C} 1 \mathrm{q}$. (C) $\mathrm{N}$-terminal sequencing of C1r p.W435R identified two cleavage sites: IQYY (new cleavage) and IIGGQ (activation cleavage) indicated by red arrows. The preceding Arg is highlighted in green. The 3D structure shows proximity between p.W435R and the new cleavage site. The two cleavage sites correspond to C1r specificity (cleavage between Arg and Ile). In the SP domain disulfide bond maintaining integrity after activation cleavage (orange) and active serine (red) are marked (PDB ID 1GPZ).

in CUB1, one variant in CUB2 or CCP1) were available for examination of $\mathrm{C} 1 \mathrm{r}$ and $\mathrm{C} 1 \mathrm{~s}$ processing and secretion by western blot with different antibodies. In cell extracts, two different $\mathrm{N}$ terminal C1r antibodies (from abcam: ab71652; ab66751) and two C1s antibodies (abcam: ab155270; ab134928) all showed non-specific additional bands and could not be used for the analyses. The C-terminal anti-C1r antibody (abcam: ab185212) showed C1r-specific bands in cell extracts and supernatants and the anti-C1s antibody (abcam: ab155270) could be used for supernatants (Figure 6). In control cells, C1r is visible as fulllength protein $(100 \mathrm{kDa})$ within cell extracts and supernatants with little evidence of activation. In contrast, patient cells showed considerable amounts of activated C1r in cell extracts in conjunction with absent or reduced $\mathrm{C} 1 \mathrm{r}$ secretion into the supernatant. Additional bands in patients 2 and 5 cell extracts (approx. $80 \mathrm{kDa})$, and control 2 and patient 5 supernatants $(\sim 115$ $\mathrm{kDa}$ ) with the C-terminal C1r antibody are either non-specific, represent non-glycosylated protein fragments or $\mathrm{C} 1 \mathrm{r}$ B-chain in complex with C1Inh. Remarkably, analyses of supernatants with an anti-C1s antibody showed large amounts of proenzyme in control supernatants but complete absence of full-length C1s in patient fibroblast supernatant. In contrast, activated C1s Achain was present in supernatants of patient fibroblasts but only in very small amounts for control fibroblasts (Figure 6D). This indicates that the presence of variant $\mathrm{C} 1 \mathrm{r}$ disturbs secretion of proenzymatic C1r WT in patients with pEDS, compatible with a gain-of-function effect.

\section{Increased Complement 1 Activation in Fibroblasts From Individuals With Periodontal EDS}

In order to assess a possible activation of the classical complement pathway caused by presence of active C1r variants we investigated the concentrations of activated complement 4 (C4a) in pEDS patient fibroblast cultures and serum samples. It should be noted that cultured skin fibroblasts express C1r, C1s, and C1Inh, but neither C1q nor C4 (22). C1q is required for specific activation of the classical complement pathway (23). C1Inh is the only known regulator of C1r and C1s (24). After addition of $\mathrm{C} 4$ to the harvested cell culture supernatant, highly significant $\mathrm{C} 4$ activation was detected in fibroblast supernatant of pEDS patients (median $40.79 \mathrm{ng} / \mathrm{ml}$, interquartile range 29.51-54.08). In contrast, values of control fibroblasts (median $4.55 \mathrm{ng} / \mathrm{ml}$, IQR 0.95-8.95) were unchanged compared to baseline in the absence of $\mathrm{C} 4$ (Figure 7A, $p=0.003$ ). The results imply that activated $\mathrm{C} 1 \mathrm{~s}$ secreted by patient fibroblasts causes C1q-independent activation of the classical complement pathway. Measurement of systemic C4a concentrations in serum samples from pEDS individuals (median $368.4 \mathrm{ng} / \mathrm{ml}$, IQR 200.5-848.2) and controls (median 223.2 ng/ml, IQR 176.5$349 ; p=0.07$ ) showed increased variation in patients, but the differences were not significant (Figure 7B). Highly elevated C4a concentrations were observed in one patient with manifest inflammatory bowel disease.

\section{DISCUSSION}

Periodontal EDS is the only known EDS type that is directly linked to the innate immune system (4). Our analyses indicate that pEDS variants cause constitutive intracellular activation of $\mathrm{C} 1$ s (and C1r) serine proteases resulting in $\mathrm{C} 4$ cleavage and local complement cascade activation, as well as other possible consequences. The organization of the complement system as an activation cascade entails an enormous 


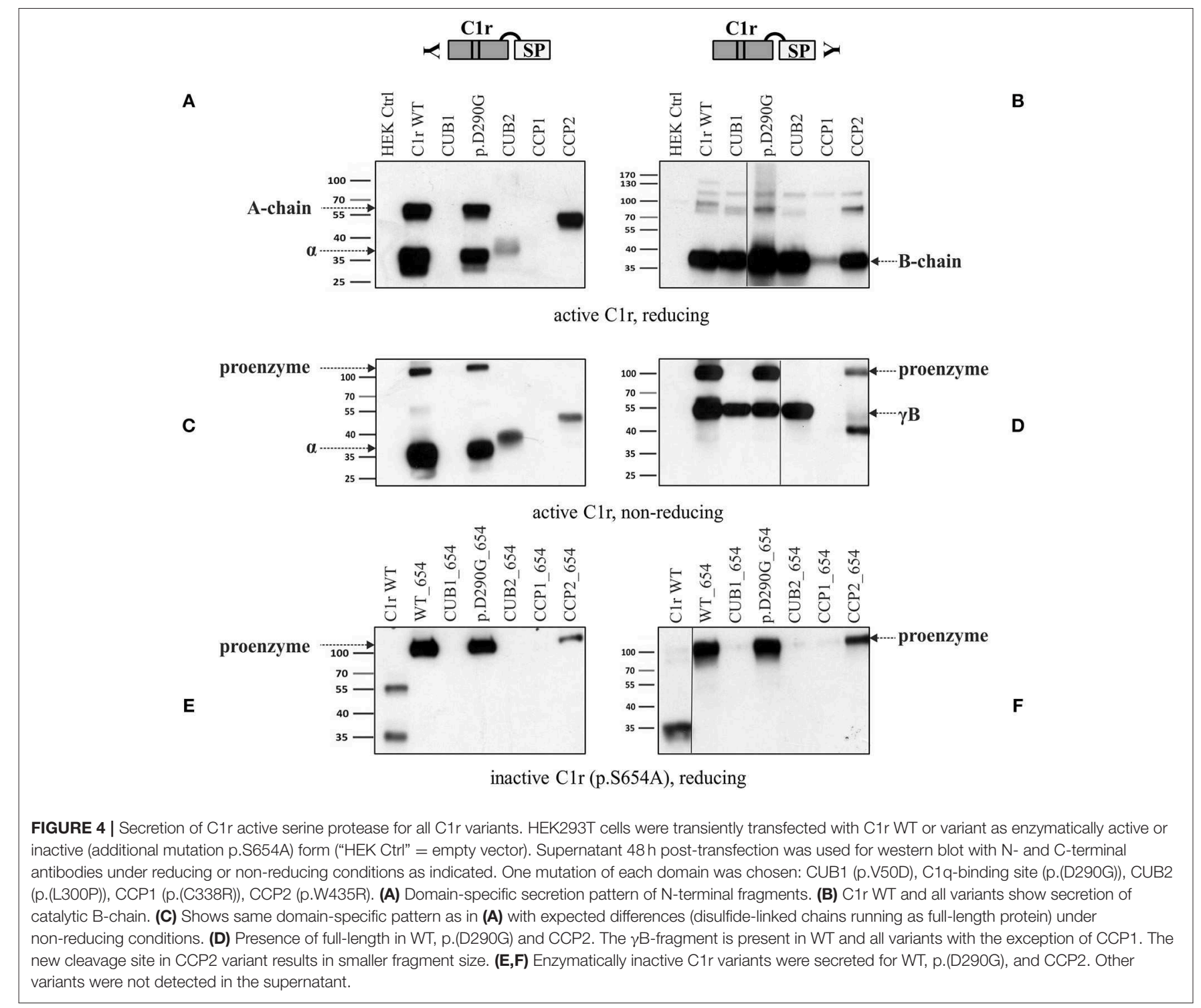

amplification capacity that therefore depends on tight regulation. Permanent immune surveillance and regulation of inflammatory pathways are essential for periodontal homeostasis. The gingival crevicular fluid that contains complement proteins is constantly secreted to keep the periodontal tissue intact (25). Even in the healthy periodontium there are low levels of inflammation mediated by innate and adaptive immune responses $(26,27)$. Studies demonstrated that C3 activation is a central element in the development of periodontitis in mice (10). Constitutive complement activation is strongly associated with periodontitis because C3-deficient mice (10) as well as C5aR1 antagonist treated mice (28) are protected against periodontitis. Complement inhibitors-such as the C3 complement inhibitor AMY-101 (29) - have been suggested as promising therapeutic options for periodontitis and have already entered clinical trials (30).

Since pEDS is a rare genetic disease, access to patient samples was limited. In addition, patient-derived fibroblasts express both C1r WT and C1r variant without possibility to distinguish between them. We therefore decided to establish an in-vitro model for a more detailed examination of intracellular processing and secretion of WT C1r and the different individual variants. Overexpression of $\mathrm{C} 1 \mathrm{r}$ in HEK293T cells differs from the physiological context as HEK293T cells do not assemble C1 complement proteins and do not produce $\mathrm{C} 1 \mathrm{Inh}$ required for the inhibition of C1r auto-cleavage. Transfected C1r WT in HEK293T cells was thus expected to show intracellular activation (Figure 2), in contrast to the proenzyme C1r WT secreted by control human-derived skin fibroblasts (Figure 6). A graphical summary is provided in Figure 8. The experiments allowed two main conclusions:

1. Different pEDS variants have very different but domainspecific effects on processing and secretion (Figure 2). There was no evidence to suggest that specific intracellular 
A

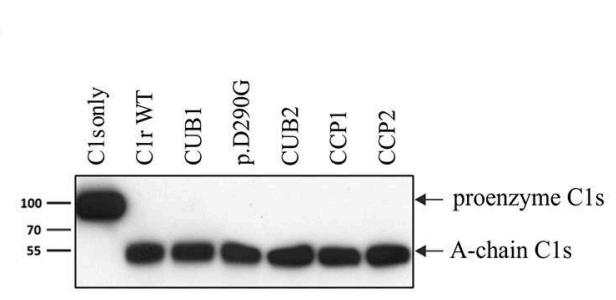

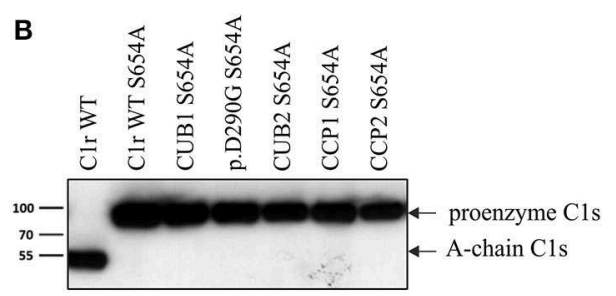
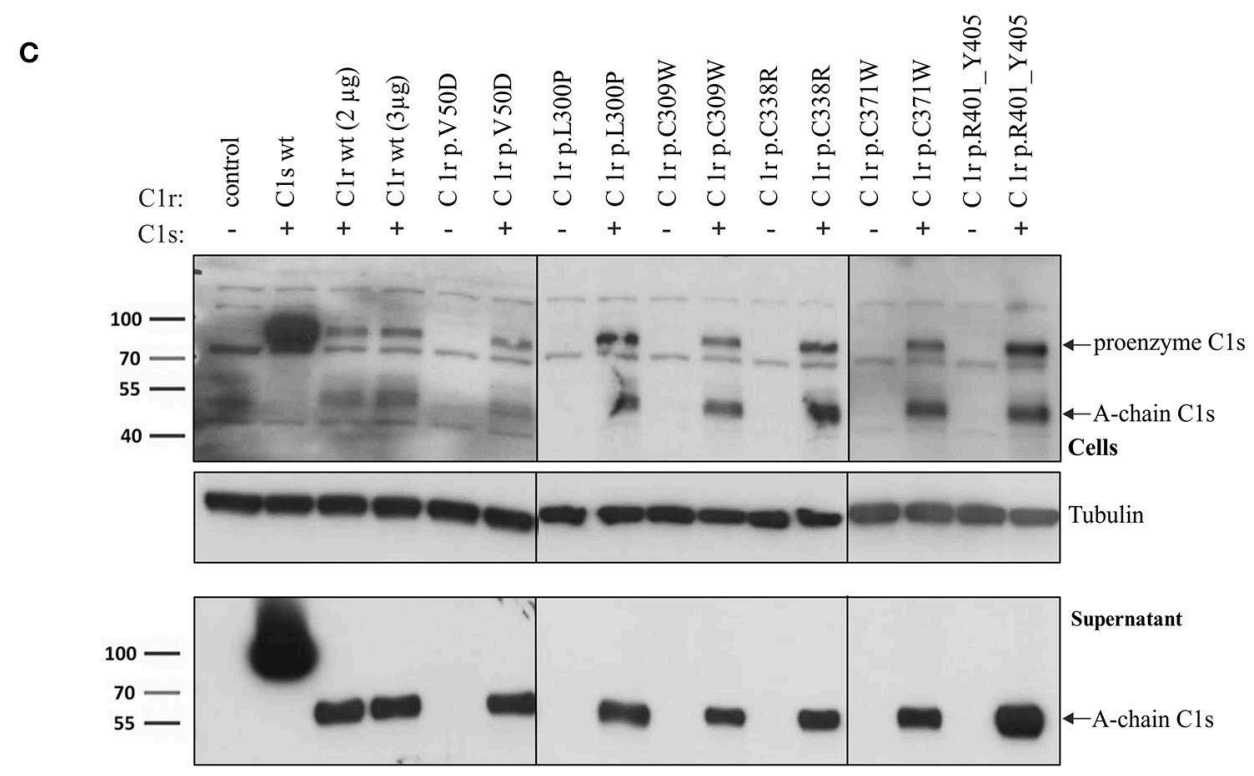

FIGURE 5 | All pEDS C1r variants retain enzymatic function toward C1s. Similarly to C1r, cleavage of proenzyme C1s (90 kDa) produces two disulfide-linked fragments: the $\mathrm{C} 1 \mathrm{~s} \mathrm{~A}$-chain ( $\mathrm{N}$-terminal, $55 \mathrm{kDa}$ ) and B-chain (C-terminal). Presence of full-length $\mathrm{C} 1 \mathrm{~s}$ and cleaved $\mathrm{C} 1 \mathrm{~s}$ (A chain) was studied with an $\mathrm{N}$-terminal anti-C1s antibody under reducing conditions. (A) HEK293T cells were transiently transfected with either C1r (WT or mutated) or C1s (WT), and supernatant was collected $48 \mathrm{~h}$ post-transfection. C1s supernatant was mixed 1:1 with supernatant from the different C1r overexpressing cells (C1r WT, CUB1, p.(D290G), CUB2, CCP1, CCP2; variants as in Figure 4), incubated for $1 \mathrm{~h}$ at $37^{\circ} \mathrm{C}$, and used for western blot analysis. C1s cleavage was observed for all cell supernatant mixtures. (B) Repeating the analysis after introduction of the inactivating mutation p.S654A into the C1r constructs prevents enzymatic cleavage of C1s in all cases. (C) C1r (WT and different variants) was overexpressed either on its own or by co-transfection with C1s WT; C1s in cell lysate (upper panel) or supernatant (lower panel) was visualized by western blot. The results show that all C1r constructs remain activity toward C1s, and that C1r-mediated C1s cleavage already occurs within the cells.

processing abnormalities or interactions of the N-terminal domains (A-chain) has specific pathogenic effects in pEDS.

2. All $C 1 R$ variants result in the production of enzymatically active serine protease (B-chain) which is secreted (Figure 4). Auto-activated $\mathrm{C} 1 \mathrm{r}$ serine protease is able to activate C1s (Figure 5).

The majority of pEDS variants showed intracellular retention (Figure 2), explaining the apparent extension of the endoplasmic reticulum after overexpression of pEDS variants in HEK293T cells (4). The C-terminal catalytic $\gamma \mathrm{B}$-chain is effectively secreted and will additionally cleave proenzymatic C1r WT that may exert pathogenic extracellular effects. Extracellular A-chain of variants was either not visualized or differed in fragment size from C1r WT on western blot, except p.(D290G) (Figure 2). A-chain is expected to be in the supernatant because $\gamma \mathrm{B}$ chain was visualized and auto-proteolytic cleavage-separating $\alpha \beta$ - from $\gamma$ B-chain (positions Arg228 and Arg296) - can only take place extracellularly in the presence of $\mathrm{Ca}^{2+}$. Thus, $\alpha \beta$-fragments are present in the supernatant but aggregate thereby hiding the C1r-antibody epitope which results in low signal intensity on western blot. Interestingly, prevention of C1r auto-activation by introduction of the active center mutation p.S654A did not interfere with secretion of uncleaved C1r WT, the C1q interaction variant p.(D290G), or the variants that introduce a new auto-activation site in the CCP2 domain. In contrast, all other pEDS variants co-expressed with p.S654A showed intracellular retention of the uncleaved protein, indicating substantial structural alterations that interfere with normal post-translational processing. It cannot be excluded that retained intracellular $\mathrm{C} 1 \mathrm{r}$ fragments have adverse effects e.g., on the processing of ECM components, but this needs further investigation.

The X-ray crystal structure of C1r CUB2 module has been solved recently, allowing identification of acidic residue D290 as a calcium ligand (31). In addition, our previous mutagenesis studies had shown that this amino acid is involved in the interaction of the C1s-C1r-C1r-C1s tetramer with C1q (21). We therefore assumed that the substitution by a non-polar glycine (p.(D290G)) would also inhibit binding of the mutated tetramer 


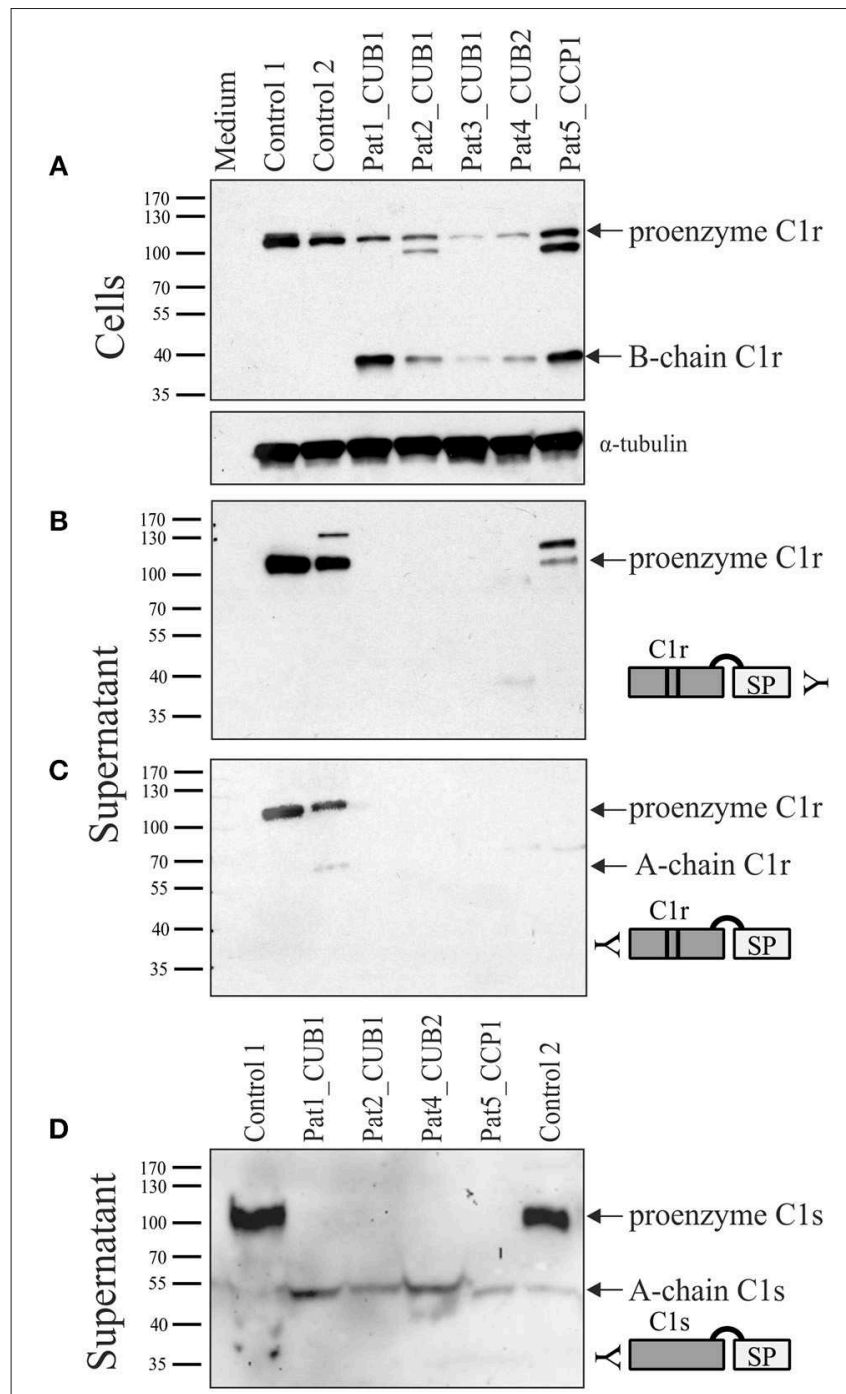

FIGURE 6 | Western blot analyses of patient and control skin fibroblast lysate and supernatant under reducing conditions. Patients 1-3 carry C1r p.V50D (CUB1), patient 4 carries C1r p.R301P (CUB2), and patient 5 carries C1r p.C309F (CCP1). Detection with C-terminal C1r antibody in cell extracts (A) and supernatant (B), and with N-terminal C1r (C) and C1s (D) antibodies in supernatant. Control cells showed uncleaved full-length $\mathrm{C} 1 \mathrm{r}$ and $\mathrm{C} 1 \mathrm{~s}$ protein both within cells (A) as well as supernatant (B-D), with little evidence of activation. In patient samples, both full-length and activated C1r (B-chain) were detected in patient cell extracts, but no C1r protein bands were visible in supernatants except at low amounts in patient 5. In contrast to controls, no full-length C1s was present in supernatants of all patient fibroblasts (D) indicating complete $\mathrm{C} 1 \mathrm{~s}$ activation caused by the presence of heterozygous $\mathrm{C} 1 \mathrm{r}$ variants. Additional bands in patients 2 and 5 cell extracts $(\sim 80 \mathrm{kDa})$, and control 2 and patient 5 supernatants $(\sim 115 \mathrm{kDa})$ with the C-terminal $\mathrm{C} 1 \mathrm{r}$ antibody are non-specific.

to $\mathrm{Clq}$, which was confirmed by SPR analyses (Figure 3). Whether the C1q binding variant p.(D290G) also triggers abnormal intra- or extracellular $\mathrm{C} 1 \mathrm{r}$ and $\mathrm{C} 1 \mathrm{~s}$ activation remains to be confirmed through fibroblast studies from pEDS patients with this variant, which were not available up to now. One possible pathomechanism of p.(D290G) may be abnormal release of enzymatically active C1r/C1s tetramer from the complement 1

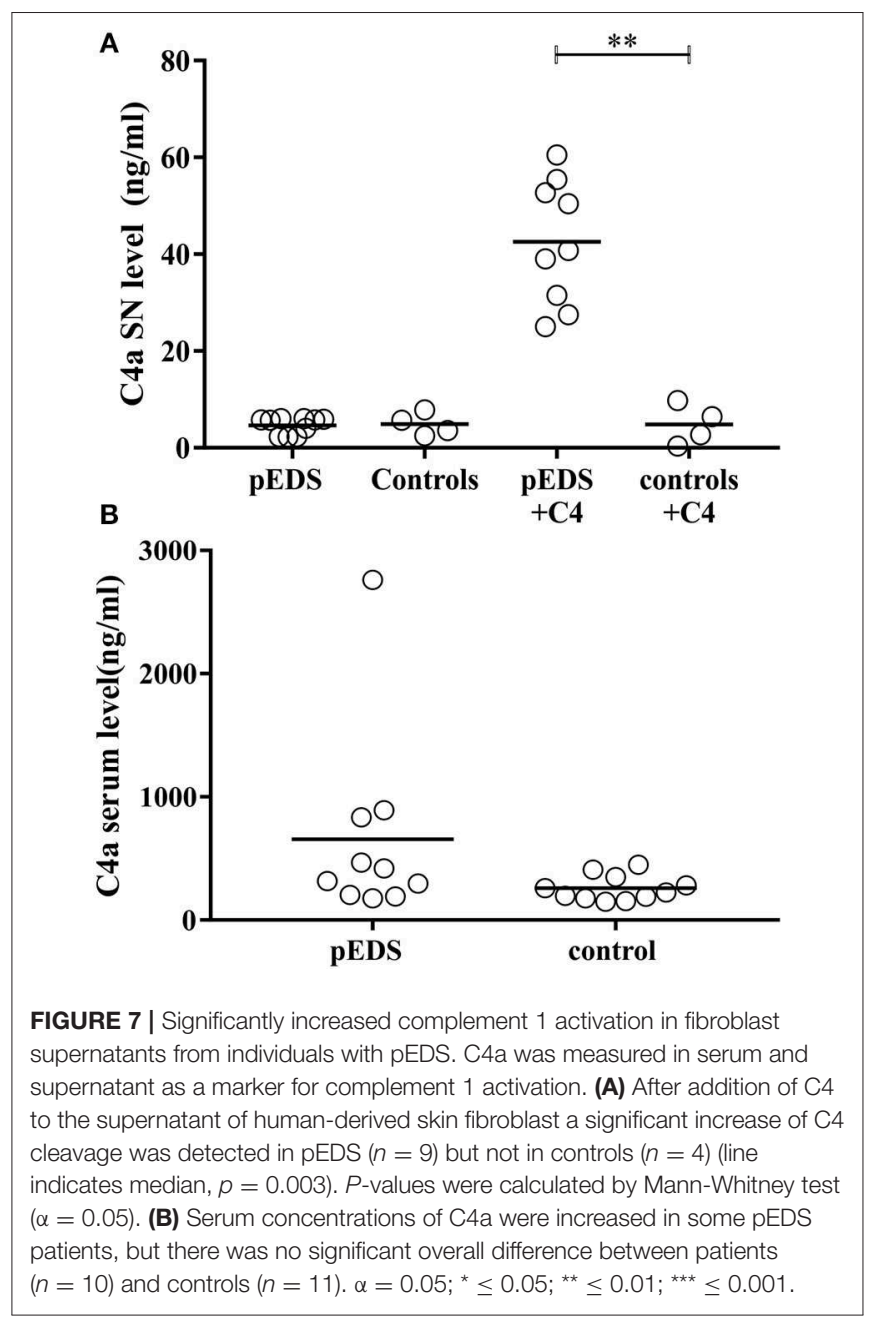

complex, but increased intracellular C1r auto-activation e.g., due to reduced C1Inh binding cannot be excluded.

C1r variant p.W435R (CCP2 domain) introduces a new cleavage site at amino acid position 401. This residue likely becomes exposed because of structural changes induced by the p.W435R variant, potentially causing partial unfolding of the CCP2 module. Destabilization of the dimeric catalytic domain of $\mathrm{C} 1 \mathrm{r}$ would result in the absence of cleavage at the usual A-chain auto-proteolysis sites. Interestingly, the second CCP2 variant [p.(Arg401_Tyr405delinsHisValIle)], which yields a similar C1r expression profile (Figure 2), results in deletion of Arg401. However, it is likely that removal of a 5-residue segment in CCP2 also results in unfolding of this domain, which might expose other potential cleavage sites at Arg or Lys residues located in the vicinity, such as Lys399. Getting a definite answer would require production and purification of the corresponding mutated C1r [p.(Arg401_Tyr405delinsHisVallle)], a time-consuming procedure due to the low amounts of mutated protein secreted.

Some mutated N-terminal C1r fragments (CUB2 variants) show a tendency to associate as dimers or to aggregate extracellularly (Figure 2 ) due to increased $\mathrm{Ca}^{2+}$ concentrations. CUB domains contain conserved $\beta$-sheets which are involved in 


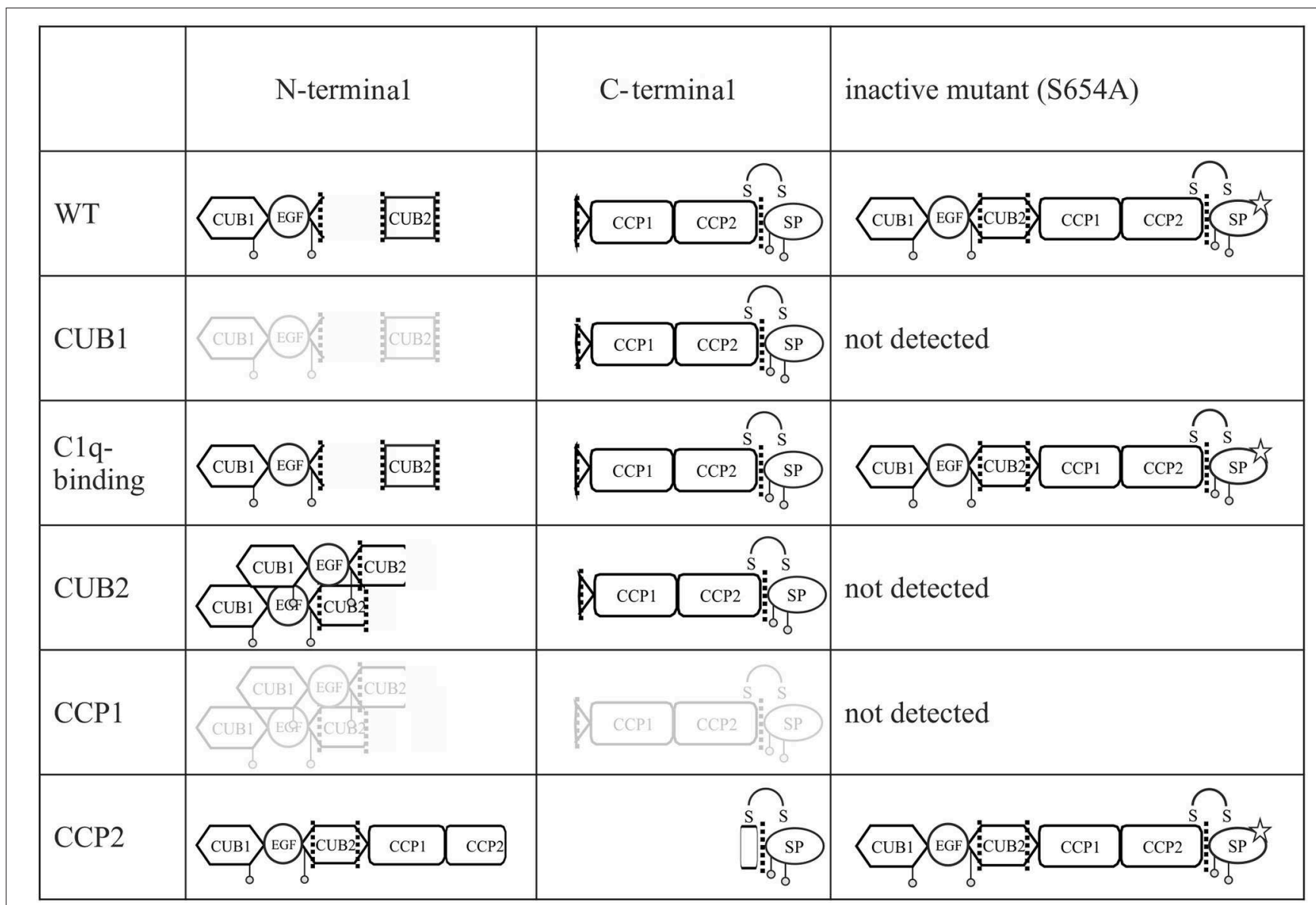

FIGURE 8 | Graphical summary of secreted fragments for all C1r variants. All fragments of enzymatic active and inactive C1r variants in the supernatant of transfected HEK293T cells and detected on western blot by $\mathrm{N}$ - and C-terminal anti-C1r antibody are presented. Fragments presented in gray color indicate faint bands on western blot. Mutation p.S654A is marked by a star. Putative aggregate formation for CUB2 mutants is indicated.

formation of aggregates $(32,33)$. It is plausible that extensive structural changes due to $\mathrm{Clr}$ variants alter the arrangement of $\beta$-sheets and facilitate aggregation, although the exact nature of this cannot be specified. As expected, western blot analysis of the putative control variant p.(R301C) showed the same pattern as WT C1r, in contrast to the pEDS variant p.R301P (Figure 2). The substitution of an arginine by a proline residue likely affects the local structure of the CUB2 domain. Covalent aggregation of mutated CUB domains combined with reduced secretion into the cell culture medium has also been noticed for the metalloprotease ADAMTS-13 (34). Moreover, the four C1r CUB2 variants with this secretion pattern are located in close proximity of the auto-proteolytic cleavage site at Arg296. While this cleavage occurs following the first auto-proteolytic cleavage at $\operatorname{Arg} 228$ upon incubation of purified serum-derived $\mathrm{C} 1 \mathrm{r}$ at $37^{\circ} \mathrm{C}(6)$, it might be favored by the variants and the resulting $\alpha \beta$-fragment would yield a band at about $40 \mathrm{kDa}$. The higher molecular weight band could correspond to dimers of this fragment. Indeed the isolated CUB1-EGF-CUB2 fragment has been shown to form dimers (31) that might be resistant to reducing conditions.
Because of the absence of C1Inh in HEK293T cells, it remained unclear whether the specific effects observed for the different variants also occur under physiological conditions. C1Inh has been shown to interact with the catalytic site of $\mathrm{C} 1 \mathrm{r}$ in the extracellular space (24). However, there have been no studies on the possible intracellular interaction of C1Inh with C1r or C1s. The three proteins are usually co-expressed in the same cell types. Considering the high auto-catalytic activity of $\mathrm{C} 1 \mathrm{r}$ it is likely that co-expression with $\mathrm{C} 1 \mathrm{Inh}$ is required for production and secretion of full-length $\mathrm{C} 1 \mathrm{r}$ (and $\mathrm{C} 1$ s together with $\mathrm{C} 1 \mathrm{r})$. Interestingly, dysfunctional C1Inh was reported in a 24 year old woman with an apparently aggressive form of periodontitis and severe angioedema restricted to the gingiva, although the molecular basis remained uncertain (35). A systematic coexpression analysis of the interaction of C1r (and its variants) with Clinh was beyond the scope of our study. Examination of available fibroblast samples from pEDS patients showed that pathogenic C1r variants trigger abnormal intracellular activation of $\mathrm{C} 1 \mathrm{r}$, whereas activation is prevented presumably by presence of the co-expressed C1Inh in control cells (Figures 6A,B). This effect should be confirmed 
by more detailed kinetic analyses for all C1r variants in future studies. Normal expression of C1Inh in patient fibroblasts was confirmed by qPCR, and genomic sequence analyses did not reveal any putative functional variants in the SERPING1 gene which codes for C1Inh (data not shown). Absent or hardly detectable fragments of $\mathrm{Clr}$ in the supernatant of patient skin fibroblasts (Figure 6C) most likely indicate intracellular retention or degradation of misfolded activated protein. In pEDS, C1r activation also triggers complete $\mathrm{C} 1 \mathrm{~s}$ activation with a lack of $\mathrm{C} 1 \mathrm{~s}$ proenzyme in the supernatant, representing a gain-of-function effect (Figure 6D). C1r-C1s interaction via N-terminal domains is not necessary for extracellular C1s activation, as observed previously for the recombinant catalytic $\gamma \mathrm{B}$-fragment of $\mathrm{Clr}(36,37)$. When complement factor $\mathrm{C} 4$ was added to the supernatant of pEDS and control fibroblast, C4 activation in the absence of external triggers was observed only for patient cells and not for control cells (Figure 7A).

The release of activated $\mathrm{C} 1 \mathrm{~s}$ by $\mathrm{C} 1 \mathrm{r} / \mathrm{C} 1 \mathrm{~s} / \mathrm{C} 1 \mathrm{Inh}$ producing cells e.g., in the periodontal region may result in local dysregulation of the complement cascade, as well as further uncontrolled cleavage of other target(s) that still need to be investigated. Numerous components or modulators of the extracellular matrix (ECM) contain CUB domains (33) and are possible interaction partners or substrates of mutated C1r or constitutively activated $\mathrm{C} 1 \mathrm{~s}$. There was no significant difference in systemic (serum) C4a concentrations between patients and controls. One patient showed very high levels of C4a, but this is well-explained by active inflammatory bowel disease in this individual. These data are in line with clinical presentation of pEDS patients who do not show symptoms of generalized systemic complement activation. This does not exclude the possibility that some clinical features of pEDS are linked to increased responsiveness to triggers of complement activation in the periodontal tissue and possibly other organs under certain conditions.

In conclusion, we report that all known $\mathrm{C} 1 \mathrm{r}$ variants causing pEDS have two complement-associated features in common: (a) they result in uncontrolled cleavage of $\mathrm{Cls}$ and thus a gain-of-function for uncontrolled protease activity against $\mathrm{C} 4$ and potentially unspecified other targets; (b) they are incapable of forming the $\mathrm{Cl}$ complex because of missing $\mathrm{Clq}$ binding site, extensive structural alterations, or missing interaction domains. pEDS thus seems to be a disorder of constitutive local complement cascade activation in the periodontal region and possible other effects on ECM proteins in organs with complement 1 expression.

\section{DATA AVAILABILITY STATEMENT}

All datasets generated for this study are included in the article/Supplementary Material.

\section{ETHICS STATEMENT}

The studies involving human participants were reviewed and approved by Ethics Committee of the Medical University Innsbruck (UN4501 and 1074/2017). The patients/participants provided their written informed consent to participate in this study.

\section{AUTHOR CONTRIBUTIONS}

RG performed the wet lab work, data analysis, and wrote the manuscript draft. IK-S contributed to the study design, examined patients, and took samples. AA supervised the wet lab work. RR performed western blot analyses of Figure 2. EB and DM contributed patient material and revised the manuscript. CG and NT contributed to study design. FD and IB performed the experiments with purified C1r variants. CG, IB, NT, and VR contributed to data analysis. CG, VR, and NT corrected the draft of the manuscript. HS contributed to data analysis of serum analyses. JZ contributed to study design, supervised and coordinated the project, and corrected the manuscript. All authors contributed to manuscript revision, read and approved the submitted version.

\section{FUNDING}

This work was supported by the Austrian Science Fund (FWF grant I 2909-B30) and by the French National Research Agency (grant ANR-16-CE91-0004-01). This work used the platforms of the Grenoble Eric-Instruct center (ISBG; UMS 3518 CNRSCEA-UJF-EMBL) with support from FRISBI (ANR-10-INSB05-02) and GRAL (ANR-10-LABX-49-01) within the Grenoble Partnership for Structural Biology (PSB).

\section{ACKNOWLEDGMENTS}

Special thanks to Mag. Brigitte Müllauer for her valuable support and practical contributions. We thank Jean-Pierre Andrieu and Guillaume Fouet for performing N-terminal sequence determination and SPR analyses, respectively. We are grateful to Robert James McKinlay Gardner (Genetic Health Queensland, Royal Brisbane and Women's Hospital, Herston, Australia) and Hannah Burns (School of Medicine, University of Queensland, Brisbane, Australia and Department of Otolaryngology Head and Neck Surgery, Lady Cilento Childrens Hospital, Brisbane, QLD, Australia) for kindly providing precious patient material. We thank the reviewers for helpful comments and suggestions during the review process. We thank all the patients and their families who contributed to the study.

\section{SUPPLEMENTARY MATERIAL}

The Supplementary Material for this article can be found online at: https://www.frontiersin.org/articles/10.3389/fimmu. 2019.02537/full\#supplementary-material

Figure S1 | Cell lysates and supernatants of HEK293T cells with C-terminal antibody. HEK293T cells were transiently transfected with C1r WT or variants ("control" = empty vector). Cell lysates (upper panel) and supernatants (lower panel) were used for western blot with an C-terminal anti-C1r antibody $48 \mathrm{~h}$ after transfection under reducing conditions. This antibody may specifically visualize the full-length $\mathrm{C} 1 \mathrm{r}(100 \mathrm{kDa})$ and the B-chain (38 kDa) after auto-activation of C1r. Little full-length $\mathrm{C} 1 \mathrm{r}$ and presence of B-chain was detected in all cell lysates. C1r WT and all C1r variants resulted in extracellular presence of B-chain. For CCP1 mutants hardly visible bands representing the B-chain were detected. 


\section{REFERENCES}

1. Brady AF, Demirdas S, Fournel-Gigleux S, Ghali N, Giunta C, KapfererSeebacher I, et al. The Ehlers-Danlos syndromes, rare types. Am J Med Genet C Semin Med Genet. (2017) 175:70-115. doi: 10.1002/ajmg.c. 31550

2. Malfait F, Francomano C, Byers P, Belmont J, Berglund B, Black J, et al. The 2017 international classification of the Ehlers-Danlos syndromes. Am J Med Genet C Semin Med Genet. (2017) 175:8-26. doi: 10.1002/ajmg.c. 31552

3. Kapferer-Seebacher I, Lundberg P, Malfait F, Zschocke J. Periodontal manifestations of Ehlers-Danlos syndromes: a systematic review. J Clin Periodontol. (2017) 44:1088-100. doi: 10.1111/jcpe.12807

4. Kapferer-Seebacher I, Pepin M, Werner R, Aitman TJ, Nordgren A, Stoiber H, et al. Periodontal Ehlers-Danlos syndrome is caused by mutations in C1R and C1S, which encode subcomponents C1r and C1s of complement. Am J Hum Genet. (2016) 99:1005-14. doi: 10.1016/j.ajhg.2016. 08.019

5. Arlaud GJ, Villiers CL, Chesne S, Colomb MG. Purified proenzyme C1r. Some characteristics of its activation and subsequent proteolytic cleavage. Biochim. Biophys. Acta. (1980) 616:116-29.

6. Arlaud GJ, Willis AC, Gagnon J. Complete amino acid sequence of the A chain of human complement-classical-pathway enzyme C1r. Biochem J. (1987) 241:711-20. doi: 10.1042/bj2410711

7. Gaboriaud C, Ling WL, Thielens NM, Bally I, Rossi V. Deciphering the fine details of $\mathrm{cl}$ assembly and activation mechanisms: "mission impossible"? Front Immunol. (2014) 5:565. doi: 10.3389/fimmu.2014. 00565

8. Gal P, Dobo J, Zavodszky P, Sim RB. Early complement proteases: C1r, C1s and MASPs. A structural insight into activation and functions. Mol Immunol. (2009) 46:2745-52. doi: 10.1016/j.molimm.2009.04.026

9. Hajishengallis G, Abe T, Maekawa T, Hajishengallis E, Lambris JD. Role of complement in host-microbe homeostasis of the periodontium. Semin Immunol. (2013) 25:65-72. doi: 10.1016/j.smim.2013. 04.004

10. Maekawa T, Abe T, Hajishengallis E, Hosur KB, DeAngelis RA, Ricklin D, et al. Genetic and intervention studies implicating complement C3 as a major target for the treatment of periodontitis. J Immunol. (2014) 192:6020-7. doi: 10.4049/jimmunol.140 0569

11. Mastellos DC, Ricklin D, Hajishengallis E, Hajishengallis G, Lambris JD. Complement therapeutics in inflammatory diseases: promising drug candidates for C3-targeted intervention. Mol Oral Microbiol. (2016) 31:3-17. doi: 10.1111/omi.12129

12. Hajishengallis G, Kajikawa T, Hajishengallis E, Maekawa T, Reis ES, Mastellos DC, et al. Complement-dependent mechanisms and interventions in periodontal disease. Front Immunol. (2019) 10:406. doi: 10.3389/fimmu.2019. 00406

13. Amano MT, Ferriani VP, Florido MP, Reis ES, Delcolli MI, Azzolini $\mathrm{AE}$, et al. Genetic analysis of complement $\mathrm{C} 1 \mathrm{~s}$ deficiency associated with systemic lupus erythematosus highlights alternative splicing of normal C1s gene. Mol Immunol. (2008) 45:1693-702. doi: 10.1016/j.molimm.2007. 09.034

14. Demirkaya E, Zhou Q, Smith CK, Ombrello MJ, Deuitch N, Tsai WL, et al. Brief report: deficiency of complement $1 \mathrm{r}$ subcomponent in early-onset systemic lupus erythematosus: the role of disease-modifying alleles in a monogenic disease. Arthritis Rheumatol. (2017) 69:1832-9. doi: 10.1002/art.40158

15. Wu YL, Brookshire BP, Verani RR, Arnett FC, Yu CY. Clinical presentations and molecular basis of complement C1r deficiency in a male AfricanAmerican patient with systemic lupus erythematosus. Lupus. (2011) 20:112634. doi: 10.1177/0961203311404914

16. Kapferer-Seebacher I, Waisfisz Q, Boesch S, Bronk M, van Tintelen P, Gizewski ER, et al. Periodontal Ehlers-Danlos syndrome is associated with leukoencephalopathy. Neurogenetics. (2018) 20:1-8. doi: 10.1007/s10048-0180560-x
17. Lek M, Karczewski KJ, Minikel EV, Samocha KE, Banks E, Fennell $\mathrm{T}$, et al. Analysis of protein-coding genetic variation in 60,706 humans. Nature. (2016) 536:285-91. doi: 10.1038/nature 19057

18. Budayova-Spano M, Grabarse W, Thielens NM, Hillen H, Lacroix M, Schmidt M, et al. Monomeric structures of the zymogen and active catalytic domain of complement protease clr: further insights into the $\mathrm{cl}$ activation mechanism. Structure. (2002) 10:1509-19. doi: 10.1016/S0969-2126(02) 00881-X

19. Bally I, Inforzato A, Dalonneau F, Stravalaci M, Bottazzi B, Gaboriaud C, et al. Interaction of $\mathrm{Clq}$ with pentraxin 3 and IgM revisited: mutational studies with recombinant C1q variants. Front Immunol. (2019) 10:461. doi: 10.3389/fimmu.2019.00461

20. Arlaud GJ, Sim RB, Duplaa AM, Colomb MG. Differential elution of Clq, $\mathrm{Clr}$ and $\mathrm{Cls}$ from human $\mathrm{Cl}$ bound to immune aggregates. Use in the rapid purification of $\mathrm{Cl}$ subcomponents. Mol Immunol. (1979) 16:445-50. doi: 10.1016/0161-5890(79)90069-5

21. Bally I, Rossi V, Lunardi T, Thielens NM, Gaboriaud C, Arlaud GJ. Identification of the Clq-binding Sites of Human $\mathrm{Clr}$ and C1s: a refined three-dimensional model of the C1 complex of complement. J Biol Chem. (2009) 284:19340-8. doi: 10.1074/jbc.M109. 004473

22. Marthandan S, Priebe S, Baumgart M, Groth M, Cellerino A, Guthke R, et al. Similarities in gene expression profiles during in vitro aging of primary human embryonic lung and foreskin fibroblasts. Biomed Res Int. (2015) 2015:731938. doi: $10.1155 / 2015 / 731938$

23. Gulati P, Lemercier C, Guc D, Lappin D, Whaley K. Regulation of the synthesis of $\mathrm{C} 1$ subcomponents and C1-inhibitor. Behring Inst Mitt. (1993):196-203.

24. Ziccardi RJ. A new role for C-1-inhibitor in homeostasis: control of activation of the first component of human complement. J Immunol. (1982) 128:2505-8.

25. Kornman KS, Page RC, Tonetti MS. The host response to the microbial challenge in periodontitis: assembling the players. Periodontol 2000. (1997) 14:33-53. doi: 10.1111/j.1600-0757.1997.tb0 0191.x

26. Dutzan N, Konkel JE, Greenwell-Wild T, Moutsopoulos NM. Characterization of the human immune cell network at the gingival barrier. Mucosal Immunol. (2016) 9:1163-72. doi: 10.1038/mi. 2015.136

27. Moutsopoulos NM, Konkel JE. Tissue-specific immunity at the oral mucosal barrier. Trends Immunol. (2018) 39:276-87. doi: 10.1016/j.it.2017. 08.005

28. Kohl J. Drug evaluation: the C5a receptor antagonist PMX-53. Curr Opin Mol Ther. (2006) 8:529-38.

29. Kajikawa T, Briones RA, Resuello RRG, Tuplano JV, Reis ES, Hajishengallis E, et al. Safety and efficacy of the complement inhibitor AMY-101 in a natural model of periodontitis in non-human primates. Mol Ther Methods Clin Dev. (2017) 6:207-15. doi: 10.1016/j.omtm.2017.08.001

30. Amyndas Pharmaceuticals SA. A Study of the C3 Complement Inhibitor AMY-101 in Adults With Gingivitis. (2018). Available online at: https:// ClinicalTrials.gov/show/NCT03694444.

31. Almitairi J, Girija UV, Furze CM, Simpson-Gray X, Badakshi F, Marshall JE, et al. Structure of the C1r-C1s interaction of the $\mathrm{C} 1$ complex of compelement activation. Proc Natl Acad Sci USA. (2018) 115:468-773. doi: 10.1073/pnas.1718709115

32. Chiti F, Dobson CM. Protein misfolding, functional amyloid, and human disease. Annu Rev Biochem. (2006) 75:333-66. doi: 10.1146/annurev.biochem.75.101304.123901

33. Gaboriaud C, Gregory-Pauron L, Teillet F, Thielens NM, Bally I, Arlaud GJ. Structure and properties of the $\mathrm{Ca}(2+)$-binding CUB domain, a widespread ligand-recognition unit involved in major biological functions. Biochem J. (2011) 439:185-93. doi: 10.1042/BJ20111027

34. Zhou Z, Yeh HC, Jing H, Wang C, Tao Z, Choi H, et al. Cysteine residues in CUB-1 domain are critical for ADAMTS13 secretion and stability. Thromb Haemost. (2011) 105:21-30. doi: 10.1160/TH10-07-0446 
35. Roberts A, Shah M, Chapple IL. C-1 esterase inhibitor dysfunction localised to the periodontal tissues: clues to the role of stress in the pathogenesis of chronic periodontitis? $J$ Clin Periodontol. (2003) 30:271-7. doi: 10.1034/j.1600-051X.2003.0 1266.x

36. Kardos J, Gal P, Szilagyi L, Thielens NM, Szilagyi K, Lorincz Z, et al. The role of the individual domains in the structure and function of the catalytic region of a modular serine protease, C1r. J Immunol. (2001) 167:5202-8. doi: 10.4049/jimmunol.167.9.5202

37. Lacroix M, Ebel C, Kardos J, Dobo J, Gal P, Zavodszky P, et al. Assembly and enzymatic properties of the catalytic domain of human complement protease C1r. J Biol Chem. (2001) 276:36233-40. doi: 10.1074/jbc.M105688200
Conflict of Interest: The authors declare that the research was conducted in the absence of any commercial or financial relationships that could be construed as a potential conflict of interest.

Copyright (C) 2019 Gröbner, Kapferer-Seebacher, Amberger, Redolfi, Dalonneau, Björck, Milnes, Bally, Rossi, Thielens, Stoiber, Gaboriaud and Zschocke. This is an open-access article distributed under the terms of the Creative Commons Attribution License (CC BY). The use, distribution or reproduction in other forums is permitted, provided the original author(s) and the copyright owner(s) are credited and that the original publication in this journal is cited, in accordance with accepted academic practice. No use, distribution or reproduction is permitted which does not comply with these terms. 